\title{
A Self-adaptive Bat Algorithm for Camera Calibration
}

\author{
Liu Xiaozhi ${ }^{1, a}$, Qi Didi ${ }^{1, b}$ \\ ${ }^{1}$ College of Information Science and Engineering, Northeastern University, Shenyang, China \\ aliuxiaozhi@mail.neu.edu.cn, bqidi9519@sina.com
}

Keywords: Camera calibration, Self-adaptive bat algorithm, Intrinsic parameters

\begin{abstract}
To obtain more accurate results of optimization calibration, an improved self-adaptive bat algorithm of camera calibration was proposed. Firstly, step parameters were set adaptively so that the objective function could avoid local minima. Secondly, the improved bat algorithms used in non-linear camera calibration did not need initial estimation values. So the proposed method could solve the problem that traditional optimization algorithms were sensitive to initial value. Furthermore, the self-adaptive bat algorithm combined with the process of camera calibration was used to optimize the camera's intrinsic parameters and the coefficient of radial distortion. Finally, the average re-projection error was analyzed, and the mean absolute error and the standard deviation were also calculated on the cases of different noise level. The experimental evaluation demonstrates that the proposed method was more efficient and accurate.
\end{abstract}

\section{Introduction}

Camera calibration is a very important and necessary technique in computer vision, medical photogrammetry, and robot vision. The relationship between 3D geometrical position of a spatial point and the corresponding point in its image is determined by the geometrical model of camera imaging. The parameters in this model are called camera parameters including intrinsic parameters and extrinsic parameters. These parameters are obtained necessarily by experiments and computation and this process is called camera calibration ${ }^{[1]}$.

A number of methods have been investigated in camera calibration. Algorithms for calibrating a pinhole camera can be primarily classified into two categories: traditional ways of calibration ${ }^{[2,3]}$ and self-calibration $^{[4-6]}$. In the first category, a well-made 3D calibration target with known geometry is needed to establish the 3D points on the object and the corresponding 2D image point on the image plane. So these methods have high accuracy. However when we need to adjust the lens constantly, these methods are not applicable. The self-calibration method, which does not need any objects of specific known dimension, or even without any calibration objects. Since there are many parameters to estimate, it doesn't always obtain reliable results. Due to their ease of use, calibration algorithms that use planar patterns have gained widespread acceptance.

To deal with those defects mentioned above in traditional algorithms, a self-adaptive calibration method on the basis of bat algorithm was proposed in this paper. The proposed method combining with camera calibration was easily operation and strong robustness.

\section{Camera Model and Parameters}

Camera calibration is the process of determining the extrinsic and intrinsic parameters of camera.

Idea Pinhole Camera Model. The existing widely-used pinhole camera model is adopted in this paper. The projection transformation process from a $3 \mathrm{D}$ point in space to a $2 \mathrm{D}$ image point on the camera plane is shown in Fig. 1.

In Fig. 1 , there is a 3D point $\mathrm{P}$, its world coordinate is $\left(x_{w}, y_{w}, z_{w}\right)$, camera coordinate is $\left(x_{c}, y_{c}, z_{c}\right)$ and pixel image coordinate is $(u, v)$.

Transformation from the pixel image coordinate to the world coordinate can be described as: 


$$
z_{c}\left[\begin{array}{c}
u \\
v \\
1
\end{array}\right]=\left[\begin{array}{ccc}
a_{u} & s & u_{0} \\
0 & a_{v} & v_{0} \\
0 & 0 & 1
\end{array}\right]\left[\begin{array}{ll}
\boldsymbol{R} & \boldsymbol{t}
\end{array}\right]\left[\begin{array}{c}
x_{w} \\
y_{w} \\
z_{w} \\
1
\end{array}\right]=\boldsymbol{K}\left[\begin{array}{lll}
\boldsymbol{r}_{1} & \boldsymbol{r}_{2} & \boldsymbol{r}_{3}
\end{array}\right] \tilde{\boldsymbol{X}}
$$

where $\boldsymbol{K}$ is the camera's intrinsic parameters matrix, $\boldsymbol{R}$ is a orthogonal rotation matrix and $\boldsymbol{t}$ is a translation vector that represent the relative rotation and translation between the world coordinate and camera coordinate, respectively. The unknown parameters in $\boldsymbol{R}$ and $\boldsymbol{t}$ are camera's extrinsic parameters. $\left(u_{0}, v_{0}\right)$ is the coordinate of the principal point; $a_{u}$ and $a_{v}$ are the scale factors in $u$ and $v$ axes, and $s$ denotes the skewness of the two image axes.

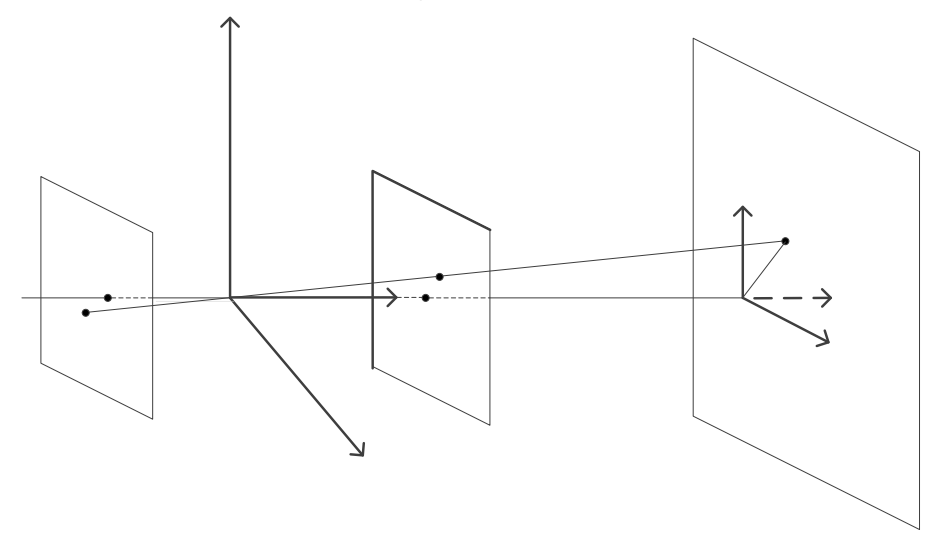

Fig. 1. The camera imaging model

According to computer vision theory, assume the model plane is on $Z=0$ of the world coordinate, then Eq. 1 can be reduced as:

$$
z_{c}\left[\begin{array}{c}
u \\
v \\
1
\end{array}\right]=\boldsymbol{K}\left[\begin{array}{llll}
\boldsymbol{r}_{1} & \boldsymbol{r}_{2} & \boldsymbol{r}_{3} & \boldsymbol{t}
\end{array}\right]\left[\begin{array}{c}
x_{w} \\
y_{w} \\
0 \\
1
\end{array}\right]=\boldsymbol{K}\left[\begin{array}{lll}
\boldsymbol{r}_{1} & \boldsymbol{r}_{2} & \boldsymbol{t}
\end{array}\right]\left[\begin{array}{c}
x_{w} \\
y_{w} \\
1
\end{array}\right]
$$

According to Eq. 2, homography matrix between the point on the image and the point on the template can be represented as:

$$
\boldsymbol{H}^{\prime}=\left[\begin{array}{lll}
\boldsymbol{h}_{1} & \boldsymbol{h}_{2} & \boldsymbol{h}_{3}
\end{array}\right]=\lambda \boldsymbol{K}\left[\begin{array}{lll}
\boldsymbol{r}_{1} & \boldsymbol{r}_{2} & \boldsymbol{t}
\end{array}\right]
$$

where $\lambda$ is an arbitrary scale factor.

Let

$$
\boldsymbol{B}=\boldsymbol{K}^{-T} \boldsymbol{K}^{-1}=\left[\begin{array}{llll}
B_{11} & B_{12} & B_{13} \\
B_{21} & B_{22} & B_{23} \\
B_{31} & B_{32} & B_{33}
\end{array}\right]=\left[\begin{array}{ccc}
\frac{1}{\alpha_{u}^{2}} & -\frac{s}{\alpha_{u}^{2} \alpha_{v}} & \frac{v_{0} s-u_{0} \alpha_{v}}{\alpha^{2} \beta} \\
-\frac{s}{\alpha_{u}^{2} \alpha_{v}} & \frac{s^{2}}{\alpha_{u}^{2} \alpha_{v}^{2}}-\frac{1}{\alpha_{v}^{2}} & -\frac{s\left(v_{0} s-u_{0} \alpha_{v}\right)}{\alpha_{u}^{2} \alpha_{v}^{2}}-\frac{v_{0}}{\alpha_{v}^{2}} \\
\frac{v_{0} s-u_{0} \alpha_{v}}{\alpha_{u}^{2} \alpha_{v}} & -\frac{s\left(v_{0} s-u_{0} \alpha_{v}\right)}{\alpha_{u}^{2} \alpha_{v}^{2}}-\frac{v_{0}}{\alpha_{v}^{2}} & \frac{\left(v_{0} s-u_{0} \alpha_{v}\right)^{2}}{\alpha_{u}^{2} \alpha_{v}^{2}}+\frac{v_{0}^{2}}{\alpha_{v}^{2}}+1
\end{array}\right]
$$

Because $\boldsymbol{B}$ is the symmetric matrix, it can be simplified as the following 6-d vector:

$\boldsymbol{b}=\left[\begin{array}{llllll}B_{11} & B_{12} & B_{22} & B_{13} & B_{23} & B_{33}\end{array}\right]$

According to [1], matrix $\boldsymbol{B}$ can be obtained. Then from Eq. 4, the least squares solution for intrinsic parameters can be solved as follows:

$$
\begin{aligned}
& u_{0}=\frac{s v_{0}}{a_{v}}-\frac{B_{13} a_{u}^{2}}{\lambda_{1}}, v_{0}=-\frac{\left(B_{12} B_{13}-B_{11} B_{23}\right)}{\left(B_{11} B_{22}-B_{12}{ }^{2}\right)}, \alpha_{u}=\sqrt{\frac{\lambda_{1}}{B_{11}}}, \alpha_{v}=\sqrt{\frac{\lambda_{1} B_{11}}{\left(B_{11} B_{22}-B_{12}^{2}\right)}} \\
& s=-\frac{B_{12} a_{u}^{2} a_{v}}{\lambda_{1}}, \lambda_{1}=B_{33}-\frac{B_{13}^{2}+v_{0}\left(B_{12} B_{13}-B_{11} B_{23}\right)}{B_{11}}
\end{aligned}
$$

According to Eq. 3, extrinsic parameters can be calculated as follows: 


$$
r_{1}=\lambda \boldsymbol{K}^{-1} \boldsymbol{h}_{1}, r_{2}=\lambda \boldsymbol{K}^{-1} \boldsymbol{h}_{2}, r_{3}=r_{1} \times r_{2}, t=\lambda \boldsymbol{K}^{-1} \boldsymbol{h}_{3}, \lambda=\frac{1}{\left\|\boldsymbol{K}^{-1} \boldsymbol{h}_{1}\right\|}=\frac{1}{\left\|\boldsymbol{K}^{-1} \boldsymbol{h}_{2}\right\|}
$$

Distortion Model. In this paper, we consider the second-order radial distortion. The relationship of ideal image coordinates and actual image coordinates can be described as:

$$
\left\{\begin{array}{l}
x=x_{d}+\delta_{x} \\
y=y_{d}+\delta_{y}
\end{array}\right.
$$

where $(x, y)$ is the ideal point image coordinates, and $\left(x_{d}, y_{d}\right)$ is the actual point image coordinates. $\delta_{x}$ and $\delta_{y}$ can be expressed as:

$$
\left\{\begin{array}{l}
\delta_{x}=\left(x_{d}-u_{0}\right)\left(k_{1} r^{2}+k_{2} r^{4}\right) \\
\delta_{y}=\left(y_{d}-v_{0}\right)\left(k_{1} r^{2}+k_{2} r^{4}\right)
\end{array}, r^{2}=x_{d}^{2}+y_{d}^{2}\right.
$$

where $k_{1}$ and $k_{2}$ are the coefficients of the radial distortion.

In this paper, the proposed self-adaptive bat algorithm is used to obtain intrinsic camera parameters and coefficients of the radial distortion simply and accuracy.

\section{Camera Calibration By Improved Self-adaptive Bat Algorithm}

Bat Algorithm. In 2010, a novel meta-heuristic optimization algorithm called the bat algorithm (BA) was proposed by Yang ${ }^{[7]}$. At present, the bat algorithm is in-depth studied by many scholars ${ }^{[8-10]}$.However bat algorithm is always falling into local optimum or lacking of search activity. So a self-adaptive bat algorithm (SABA) is proposed to overcome these shortcomings. We can use the SABA to solving camera parameters.

Improved Self-adaptive Bat Algorithm. In order to enhance the search ability of this algorithm, a new self-adaptive bat algorithm (SABA) is proposed in this paper. The process of the SABA for camera calibration can be described as follows.

Self-adaptive bat algorithm is based on three ideal rules:

i) Bat uses echolocation to sense the distance and differentiate between food/prey and background barriers even in the darkness;

ii) Bats fly randomly to search prey with position $\left(x_{i}\right)$, velocity $\left(v_{i}\right)$, frequency $\left(f_{i}\right)$, loudness $\left(A_{i}\right)$ and rate of pulse emission $\left(r_{i}\right)$;

iii) The loudness varies from a maximum loudness ( $\left.A_{\max }\right)$ to the minimum loudness $\left(A_{\min }\right)$.

In SABA, each bat can be expressed as:

$x_{i}=\left(x_{i 1}, \mathrm{z}_{i 2} \ldots x_{i P}\right) \quad i=(1,2 \ldots N)$

Camera's parameters $\left(a_{u}, a_{v}, s, u_{0}, v_{0}, k_{1}, k_{2}\right)$ can be expressed as $\left(x_{i 1}, z_{i 2} \ldots x_{i P}\right)$.

Initialization. Set the generation counter $t=1$; initialize the population of $\mathrm{N}=50$ bats randomly and each bat have $P=7$ vector which is corresponding to a potential solution to the given problem.

For camera calibration problems, the SABA treats potential solutions as virtual micro bats whose velocities $v_{i}$, positions $x_{i}$ and frequencies $q_{i}$ can be updated as follows:

$$
\begin{aligned}
& q_{i}=q_{\min }+\left(q_{\max }-q_{\min }\right) \theta_{i} \quad \theta_{i}=\frac{\left(f_{i}-f_{\min }\right) \zeta}{f_{i}} \\
& v_{i}^{t}=v_{i}^{t-1}+\left(x^{*}-x_{i}^{t-1}\right) q_{i} \quad x_{i}^{t}=x_{i}^{t-1}+v_{i}^{t}
\end{aligned}
$$

Where $f_{i}$ denotes the fitness function and $\zeta$ denotes the constant. The initial position $x_{i}^{0}$ is a random vector distributed uniformly within $\left[x_{i, \min }, x_{i, \max }\right]$. The initial velocity $v_{i}^{0}$ is a vector consisting of all zeros. Here $x^{*}$ is the current global best location (solution) which is located after comparing all the solutions among all the $n$ bats. Generally speaking, depending on the domain size of the problem of interest, the frequency $q$ is assigned to $q_{\min }=0$ and $q_{\max }=100$ in practical implementation.

Calculate Fitness Function Value. For bat algorithm, we need to define a fitness function to assess the bat's position. Only in this way can we select the best result for camera parameters.

We define the fitness function as follows: 


$$
f(x)=\frac{1}{N} \sum_{i=1}^{N} \sum_{j}^{c}\left\|m_{i j}-\hat{m}_{i j}\right\|
$$

Where $m_{i j}$ is the real pixel coordinate. $\hat{m}_{i j}$ is the pixel coordinate calculated by Eq. 2. From Eq. 14, we can find out $f_{\min }$. So the best result $x^{*}$ is $f_{\min }$.

Update and Iterative. Assume that $\delta \in[0-1]$ is a random number. If $\delta>r_{i}$, select a solution among the best solutions and generate a local solution around the selected best solution according to (15). If $\delta \leq r_{i}$, generate a new solution by flying randomly.

$x_{i, \text { new }}^{t}=x_{i, \text { old }}^{t}+\left(x^{*}-x_{i, \text { old }}^{t}\right)$

If $\delta<A_{i}$ and $f\left(x_{\text {new }}\right)<f\left(x_{i}\right)$, accept the new solution, and increase $r_{i}$ and reduce $A_{i}$ as follows:

$$
A_{i}^{t+1}=\frac{\left(f_{i}-f_{\min }\right) \zeta_{1}}{f_{i}} A_{i}^{t} \quad r_{i}^{t+1}=r_{i}^{0}\left[1-\exp \left(-\delta_{i} t\right)\right] \quad \delta_{i}=\frac{\left(f_{i}-f_{\min }\right) \zeta_{2}}{f_{i}} \text { ? }
$$

The initial loudness $A_{i}^{0}$ is a random number uniformly distributed within [1, 2]. The initial pulse emission rate $r_{i}^{0}$ is a random number uniformly distributed within $[0,1] . \zeta_{1}$ and $\zeta_{2}$ are constants.

Both $A_{i}^{t}$ and $r_{i}^{t}$ are set to the big and small values in the first iteration and then decreased and increased slowly during the optimization process to find the prey and raise the attack, respectively [10]. $r_{i}^{0}$ is the emission pulse rate at time $\mathrm{t}=0$. For any $0<\alpha<1$ and $\mu>0$, we have:

$$
A_{i}^{t} \rightarrow 0 \text { as } t \rightarrow \infty ; r_{i}^{t} \rightarrow 0 \text { as } t \rightarrow \infty
$$

Obtain the Optimal Value. Rank the bats/solutions and find the current best set ${ }^{*}$. The number of iterations adds 1 .

Completing one iteration and determine whether reached the maximum number of iterations or required accuracy. If meet the termination conditions it will output the optimal value $x^{*}$. Otherwise, return to update and iterative process described in Update and Iterative.

\section{Experiment and Analysis}

We use Genie M1024 CCD capturing standard calibration images. With the proposed calibration method and calibration steps mentioned above, we compiled the optimization algorithm program in Matlab 2011b.

To verify the effectiveness of our optimized method in terms of camera calibration, we have tested 3 calibration images which taken from different angles of calibration board. The three pictures are shown in Fig. 2.

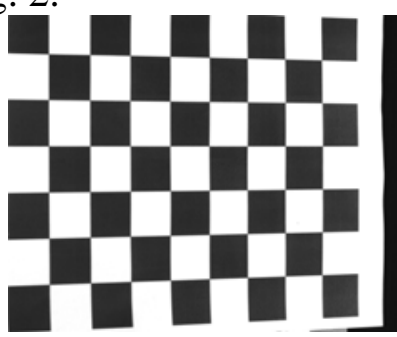

(a)

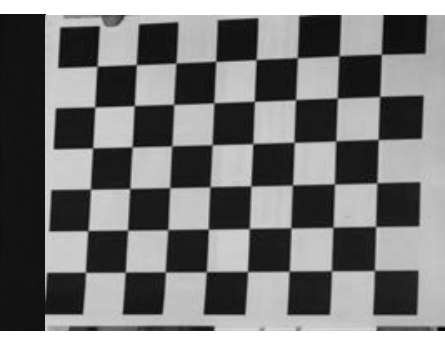

(b)

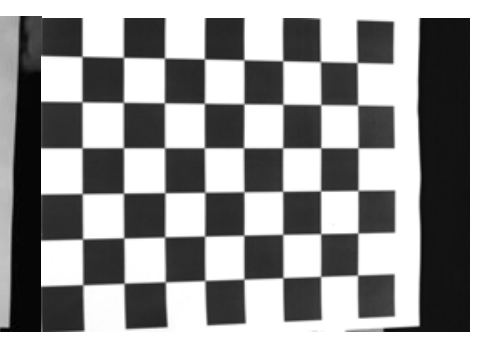

(c)

Fig. 2. The acquired images at three different locations

Set the maximal count of iteration times $\operatorname{Tmax}=3000$. The results of camera internal and external parameters are listed in Table 2 and Table 3.

Table 2. Calibration Result of Intrinsic Parameters

\begin{tabular}{ccccccc}
\hline \hline$a_{u}$ & $a_{v}$ & $s$ & $u_{0}$ & $v_{0}$ & $k_{1}$ & $k_{2}$ \\
\hline 5623.85 & 5605.39 & 57.30 & 566.88 & 390.21 & -0.466 & -0.9085
\end{tabular}

To verify the accuracy of our optimized method in terms of camera calibration, this paper randomly drawn from the third picture with 9 group testing points and compared the corresponding optimized re-projection values. The difference values are listed in Table 4. 
Table 4 shows that the re-projection errors are within 1 pixels and the mean value is $(0.4730+0.5094) / 2=0.4912$. This result shows that the calibration accuracy of the proposed method is very high.

Table 3. Calibration Result of Extrinsic Parameters

\begin{tabular}{|c|c|c|c|c|c|c|c|c|c|}
\hline & \multicolumn{3}{|c|}{ Fig.(a) } & \multicolumn{3}{|c|}{ Fig.(b) } & \multicolumn{3}{|c|}{ Fig.(c) } \\
\hline \multirow{3}{*}{$\boldsymbol{R}$} & 0.8865 & -0.0316 & 0.49017 & 0.9280 & -0.0482 & 0.3695 & 0.8833 & -0.0127 & -0.4686 \\
\hline & 0.0052 & 0.9989 & 0.0485 & -0.0222 & 0.9827 & 0.1837 & -0.0112 & 0.9988 & -0.0481 \\
\hline & -0.5001 & -0.0369 & 0.8745 & -0.3720 & -0.1787 & 0.9109 & 0.4687 & 0.0477 & 0.8821 \\
\hline$t$ & {$[-14.9987$} & -11.0097 & 158.8012] & {$[-14.5907$} & -10.7320 & 157.0259] & {$[-14.3120$} & -11.0002 & $140.4811]$ \\
\hline
\end{tabular}

Table 4. Comparison between measured values and optimized values of re-projection (pixels)

\begin{tabular}{c|c|c}
\hline \hline actual image coordinates & computed image coordinates & difference value \\
\hline$(22.6665,540.3332)$ & $(22.7602,540.9512)$ & $(0.0937,0.618)$ \\
\hline$(118.7936,203.5971)$ & $(118.1235,204.7592)$ & $(0.6701,1.1621)$ \\
\hline$(216.8336,317.5536)$ & $(216.3984,317.8963)$ & $(0.4352,0.3427)$ \\
\hline$(314.8621,647.7314)$ & $(314.4523,648.6235)$ & $(0.4368,0.8921)$ \\
\hline$(409.9510,427.1547)$ & $(410.2547,427.7852)$ & $(0.3037,0.6305)$ \\
\hline$(503.8552,535.5762)$ & $(503.1549,535.1963)$ & $(0.7003,0.3799)$ \\
\hline$(682.8138,428.5613)$ & $(682.5521,428.1336)$ & $(0.2617,0.4277)$ \\
\hline$(769.8613,114.9455)$ & $(770.3259,114.5234)$ & $(0.4646,0.4221)$ \\
\hline$(854.1831,16.4275)$ & $(854.5429,16.2675)$ & $(0.3598,0.16)$ \\
\hline-- & -- & $(0.4730,0.5094)$ \\
\hline \hline
\end{tabular}

In order to test the effectiveness of proposed method, we compare the re-projection error of Zhang method [1] and the QPSO method. We can obtain that the re-projection error of Zhang method is 1.18901, QPSO method is 0.7616 and the proposed method in this paper is 0.4912 . This indicated that the proposed method has high precision and the algorithm has feasibility.

To evaluate the robustness of the proposed method, we add Gaussian noise to the feature points and compute average absolute error and relative error in the current noise levels. The results can be shown in Fig. 3.
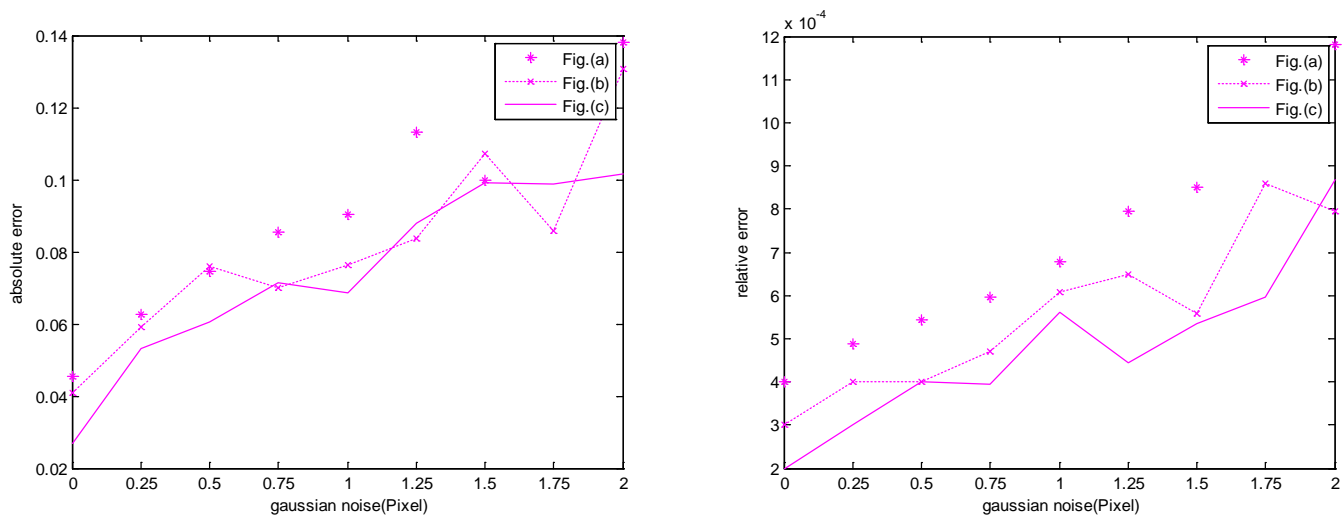

Fig. 3. The absolute error and relative error of world coordinates

As shown in Fig. 3, with the increase of the noise, the error results more and more large. However it still maintained in a very small error range. 


\section{Conclusion}

This paper has presented a more accurate and general optimization method which called SABA for a CCD camera. SABA overcomes shortcomings in traditional optimization algorithms. Experiment shows that applying SABA into nonlinear camera calibration should be a right and effective method.

\section{References}

[1] Z.Zhang, A flexible new technique for camera calibration, IEEE Trans. on Pattern Analysis and Machine Intelligence, Vol.22, No.11, 1330-1334, 2000.

[2] B Hallert, Notes on calibration of cameras and photographs in photogrammetry, Photogrametria, Vol.23, 163-178, 1968.

[3] Faig W, Calibration of close range photogrammetric systems: mathematical formulation, Photogrammetric Eng. Remote sensing, Vol.41, No.12, 1479-1486, 1975.

[4] Ma S D, A self-calibration of stationary cameras. International Journal of Computer Vision, Vol.22, No. 1, 5-23, 1997.

[5] Pollefeys M, Van Gool L, Self-calibration from the absolute conic on the plane at infinity, IEEE Trans. on Computer Analysis of Image and Pattern, Lecture Notes in Computer Science, Spring-Verlag, 1296, 175-182, 1997.

[6] Agapito L D, Hartley R L, Hayman E, Linear self-calibration of a rotating and zooming camera, Proceedings of Computer Vision and Pattern Recognition, 15-21, 1999.

[7] Yang, Xin-She, A new metaheuristic bat-inspired algorithm. Proceedings of nature inspired cooperative strategies for optimization. Springer Berlin Heidelberg, 65-74, 2010.

[8] Taher, Niknam, Rasoul, Azizipanah- Abarghooee, Mohsen Zare, and Bahman Bahmani-Firouzi, Reserve Constrained Dynamic Environmental/Economic Dispatch: A New Multiobjective Self-Adaptive Learning Bat Algorithm. IEEE trans. on Systems Journal, Vol. 7, No. 4, 763 - 776, 2013.

[9] Nien-Che Yang, Minh-Duy Le. Multi-objective bat lgorithm with time-varying inertia weights for optimal design of passive power filters set. IET Generation, Transmission \& Distribution . Vol.9, No. 7, 644-654, 2015

[10] Niknam,T, Bavafa. F, Azizipanah-Abarghooee, R, New self-adaptive bat-inspired algorithm for unit commitment problem, IEEE trans. on Science, Measurement \& Technology, Vol.8, No.6, 505-517,2014. 